\title{
Regards
}

\section{Jean-Marie Legay, du bon usage de la biométrie}

\section{Richard Tomassone}

Biométricien, Institut national agronomique, 75005 Paris, France

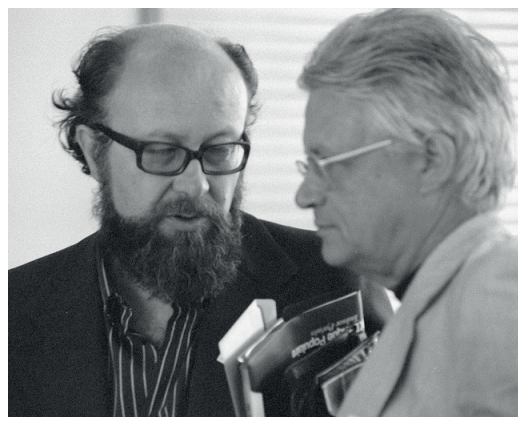

Richard Tomassone et Jean-Marie Legay (à droite de la photo) en 1982 à Toulouse lors de la $\mathrm{XI}^{\mathrm{e}}$ conférence internationale de biométrie. (C) D.R.

Jusqu'en 1970, mes relations professionnelles étaient limitées au cercle proche des chercheurs de l'Inra, surtout dans le domaine forestier, à quelques chercheurs du CNRS en écologie en poste à Montpellier et à des universitaires nancéens spécialistes de la statistique théorique. Quelques collègues européens biométriciens complétaient ce cercle.

À l'occasion d'une mutation au centre de recherches zootechniques de Jouy-en-Josas, j'eus pour mission et objectif la création d'une équipe de biométrie dont les membres devaient être ouverts à toutes collaborations avec les chercheurs de l'Inra. Ma direction ne m'avait pas demandé de privilégier un domaine particulier de recherche pour ces collaborations. De plus, la biométrie était un mot bien commode pour couvrir tout ce qui faisait appel à un outil mathématique avec de fortes doses de calcul électronique : 1 'outil était surtout celui de la statistique ; le calcul électronique en était à ses balbutiements avec un ordinateur implanté à Jouy-en-Josas et déjà très puissant pour l'époque.

J'ai donc commencé par faire un « tour de France » des différents biométriciens : ceux qui revendiquaient ce nom

\footnotetext{
Auteur correspondant : rr.tomassone@orange.fr

1 Voir aussi, dans ce numéro et le suivant, les autres hommages à Jean-Marie Legay.
}

étaient rares et, par conséquent, ce tour fut vite fait. Pourtant, beaucoup faisaient de la biométrie, mais ressentaient une sorte de honte à l'afficher, comme si cette activité se faisait sans en parler ! C'est à cette occasion que j'ai connu Jean-Marie Legay ${ }^{1}$. Dès notre première discussion, nous avons vu que, malgré nos parcours scolaires et professionnels différents, nous avions une vision très voisine de l'insertion de la biométrie dans tous les contextes de la biologie et, par conséquent, de l'agronomie.

Pour lui comme pour moi, ce n'était pas une discipline nouvelle mais une manière rigoureuse de traiter des problèmes pluridisciplinaires. C'était une démarche « humble» pour aborder des questions complexes. Par humble, nous entendions tous les deux qu'il fallait $\mathrm{d}$ 'abord écouter notre interlocuteur et nous demander ensuite si, dans notre panoplie des techniques (méthodes, outils ou modèles selon le vocabulaire compréhensible par notre interlocuteur), nous pourrions trouver le vêtement le plus adapté. Si ce vêtement existait, nous le proposions, même s'il ne nous apportait pas davantage de connaissances en biométrie. Le simple fait qu'il s'appliquât à une situation nouvelle était en soi un instrument utile pour faire avancer la recherche. Mais, si aucun vêtement ne s'ajustait correctement, alors nous nous réservions le droit de faire appel à toutes les ressources des outils formels (les mathématiques bien sûr, mais aussi la logique ou tout autre outil) pour le mettre au point.

Dans un monde scientifique où seul un résultat original est pris en compte, nous touchions du doigt la difficulté à faire admettre un tel point de vue. Un biométricien est-il un chercheur à part entière ou n'est-il qu'un prestataire de services ? Pour un biologiste, seul le résultat biologique est important ; l'analyse critique de l'outil biométrique employé ne l'intéresse généralement pas. Son attitude le conduit, à la limite, à faire une confiance absolue à un logiciel commercialisé sans qu'il soit sûr qu'un contrôle scientifique suffisant ait été réalisé. Les 
revues qui publient ses résultats veulent généralement limiter au maximum tout formalisme ou même toute réflexion sur l'outil qui a permis de réaliser le travail du biologiste. Pour un mathématicien, sans théorème nouveau, le statut de chercheur n'existe pas. L'attitude de tout biométricien le conduit à être exclu de ce statut. Comment conseiller à un jeune de choisir cette voie? Nous allions retrouver cette question de façon récurrente pendant la trentaine d'années qui ont suivi nos premières rencontres. Mais cette convergence de vue dans la fonction que nous attribuions à la biométrie a scellé, d'une certaine façon, le morceau de destin scientifique commun que nous avons eu.

La suite de nos rapports professionnels allait de soi : quand j'ai eu besoin de biométriciens dans les conseils du département de biométrie que je dirigeais, c'est à JeanMarie Legay que je faisais appel en priorité. Quand il voulait un membre de jury de thèse, il pensait à moi, sachant que je ne serais pas le diable mathématicien qui effraierait inutilement le doctorant qui, s'il a utilisé des outils biométriques, a sûrement oublié les mathématiques de l'époque proche où il passait son baccalauréat. Devenu professeur à l'Institut national agronomique,j'ai continué à faire appel à lui dans nos jurys : il venait équilibrer les jugements des mathématiciens plus théoriciens indispensables à leur constitution.

Mais ces activités que je qualifierais de parallèles ont été complétées par d'autres, plus communes. Tout d'abord, à la section française de la Société internationale de biométrie. Cette société savante internationale qui compte plus de 6000 membres à travers le monde est formée de sections régionales et quelquefois nationales, comme la section française. Dans les années 1970, elle était dirigée par deux anciens polytechniciens œuvrant dans le domaine de la recherche médicale. Comme dans beaucoup de sociétés savantes, l'activité est liée à l'enthousiasme de son président et de son secrétaire ; s'y consacrer use leur énergie. Mais, il est souvent difficile de trouver un autre couple et de lui passer le relais. Nous l'avons pris : un binôme biologiste-agronome remplaçait un binôme de médecins. Et nous avons «tenu » une dizaine d'années. Nous avons pu lancer une collection d'ouvrages «Biométrie et... » qui a connu un réel succès; cette collection reprenait les exposés présentés lors des journées annuelles de la Société. Nous avions pris le soin d'être très rigoureux dans la sélection des textes afin que les auteurs puissent en faire état dans leurs travaux. En 1977, le premier ouvrage Biométrie et Écologie ${ }^{2}$ a même dû être réédité après avoir été tiré une première fois à 500 exemplaires. Nous avons ensuite passé le relais à un autre binôme, à nouveau des médecins.

2 Legay, J.M., Tomassone, R. (Eds), 1977. Biométrie et Écologie, Paris, Société française de biométrie.
Cette activité fut l'occasion de longues discussions sur l'importance des sociétés savantes dans le domaine scientifique et technique. Jean-Marie Legay pensait qu'elles étaient un essentiel contre-pouvoir aux instances officielles et aux lobbys et qu'il fallait trouver les moyens de les protéger de ces lobbys pour qu'elles puissent garder leur indépendance de pensée. On pouvait imaginer qu'elles puissent jouer un rôle dans les domaines où une expertise scientifique s'impose. Cette idée est toujours d'actualité quand, actuellement, nous sommes confrontés aux problèmes liés à la pollution, à la toxicologie ou à l'utilité de certains médicaments. Nombre d'enquêtes dites «d'utilité publique » pour lesquelles les citoyens appelés à donner un avis n'ont aucune compétence technique et a fortiori aucun moyen d'action sont des « leurres démocratiques »; elles pourraient être confiées, pour partie, à des sociétés savantes indépendantes. Encore faudrait-il que celles-ci puissent ne pas être «sous influence ». Nous en avons longuement discuté lorsque, devenu maire de la petite commune rurale où je réside, j'ai été confronté à ce type de situation.

Nous connaissant mieux, nous avons participé ensemble, pendant de nombreuses années, à une commission multidisciplinaire de la Délégation générale à la recherche scientifique et technique, plus connue sous son sigle DGRST. Nous avons pu ainsi collaborer à des travaux aussi variés que l'étude de la bilharziose ou des groupes sanguins des Pygmées Aka. Chaque étude nous permettait de mettre en relation des disciplines différentes, médecine et linguistique, par exemple. Notre intervention a, quelquefois, permis la collaboration de chercheurs et la publication en commun de leurs résultats, qu'ils auraient pu, par individualisme, conserver jalousement pour eux.

Enfin, par deux fois, Jean-Marie Legay m'a demandé de participer aux travaux de la commission «Écologie » du CNRS. Grâce à lui, j'ai pu découvrir de l'intérieur les problèmes et le fonctionnement du plus grand organisme de recherche français. Je ne pense pas avoir apporté beaucoup, mais je crois avoir fait mûrir ma propre expérience, ce qui m'a permis ultérieurement de mieux comprendre les questions d'organisation du travail scientifique de manière beaucoup plus sereine.

L'ensemble de ces activités l'a conduit à me suggérer de prendre la direction du laboratoire de biométrie et de biologie évolutive à Lyon (de « son » laboratoire, devraisje écrire) pendant quelques années. Cette marque de confiance m'a profondément touché. Ensuite, nous avons mis de l'ordre dans nos travaux en publiant, enfin, un article commun ${ }^{3}$. Ce fut l'occasion de retourner au travail de base du chercheur. Nous avons écrit cet article en

3 Legay, J.-M., Tomassone, R., 1999. La comparaison de régressions orthogonales, Revue de Statistique Appliquée, XLVII, 2, 81-101. 
français. Dans le contexte scientifique actuel, ce fut un choix "politique » : nous pensions tous deux qu'il est important de publier dans sa langue maternelle. D'abord, parce que dans la majorité des cas, on y exprime mieux la subtilité de sa pensée que dans toute autre langue; ensuite parce que le lecteur français la comprend, lui aussi, beaucoup mieux!
Voilà, un court bilan de l'histoire de nos relations. J'ai côtoyé beaucoup de gens dans ma vie professionnelle : Jean-Marie Legay est un de ceux qui m'ont le plus marqué. J'avais la plus grande admiration pour l'homme, sa vaste culture et son intégrité exemplaire ; je suis heureux d'avoir croisé son chemin. Un détail pour finir : nous nous sommes toujours vouvoyés, alors que j'ai tendance à tutoyer rapidement mes interlocuteurs. Pourquoi ? 